\title{
Les cas bagatelles ne sont pas des bagatelles
}

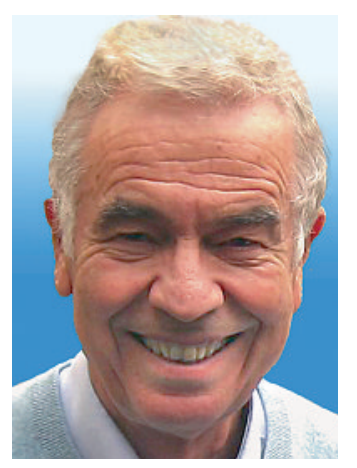

Hans Stalder
En février de cette année, une publication dans le Swiss Medical Weekly SMW [1] a montré que dans le Canton de Genève une personne sur sept renonce à des soins pour des raisons économiques. Il s'agit surtout de soins dentaires, cependant les consultations médicales, la médication, des appareils et même les interventions chirurgicales sont également concernés. Ce problème touche particulièrement les personnes avec les revenus les plus faibles et celles avec des comorbidités cardiovasculaires, donc celles qui ont le plus besoin d'être prises en charge étant d'avantage à risque de morbidité et mortalité.

Un travail antérieur [2] avait déjà révélé que 7,5\% de patients âgées et hospitalisés ont renoncé auparavant à consulter pour des raisons économiques.

Donc moins de consultations: ne faut-il pas s'en réjouir? Ces études ne montrent-elles pas que le système suisse avec franchise et participation aux frais de la santé fonctionne? Ne s'agit-il pas d'une volonté politique pour éviter les «cas bagatelles»?

\section{Une personne sur sept renonce à des soins pour des raisons économiques.}

L'idée de diminuer les coûts de santé par une participation financière des patients aux consultations a eu un appui scientifique par une grande étude de la RAND-corporation dans les années 70 [3]. Elle paraît économiquement valide et avoir du bon sens: si' j'obtiens quelque chose gratuitement, j'ai tendance à abuser. C'est ce que les anglo-saxons appellent le moral hazard. Ou autrement dit: si je suis vraiment malade, je consulte, même si je dois payer.

Cependant le système de santé est complexe et des interventions linéaires et au premier abord logiques ont quelquefois des effets inattendus.

Tout d'abord, si on demande à des collègues généralistes, s'ils sont surchargés par des bagatelles, ils ne s'en plaignent en général pas, à l'exception des arrêts de travail demandés par les employeurs. Souvent une bagatelle se révèle comme telle seulement après la consultation. De plus, chaque consultation permet des interventions de prévention (prise de tension artérielle, discussion sur l'abus de nicotine lors d'un rhume, sur l'alcool lors d'épigastralgies, etc.). Enfin, des dites «bagatelles» - rhume et lombalgies - sont économiquement lourdes en causant la majorité des absences au travail.

Par ailleurs, plusieurs études ont montré, comme l'article susmentionné [1], que ce sont souvent des médicaments essentiels qui sont arrêtés en premier quand il y a une participation au frais. Mais le plus troublant est cependant que des études plus détaillées et consécutives à l'étude RAND démontrent qu'une participation financière nouvellement introduite ou accrue, qui fait donc diminuer le nombre des consultations ambulatoires, va augmenter le nombre et les journées d'hospitalisations chez certaines personnes vulnérables - personnes âgées, atteintes de maladies chroniques, personnes économiques faibles et moins éduquées (pour la littérature, voir [4]). Donc à la place de diminuer, les frais de santé globaux augmentent dans cette population.

Les conclusions sont assez fâcheuses pour ceux qui veulent éliminer les «cas bagatelles» des nos activités ambulatoires par le moyen d'une participation des patients aux frais: S'il est vrai qu'elle diminue les coûts chez ceux qui n'ont guère besoin de soins, soit chez les jeunes fortunés, qui sont en bonne santé chez ce groupe seul les mesures de prévention sont concernés - cette même participation aux frais augmente les coûts de santé chez les aînés qui souffrent de maladies chroniques. Et ce sont justement ceux qui coûtent déjà le plus cher au système de santé!

Hans Stalder*

\section{Références}

1 Wolff H, Gaspoz JM, Guessous I. Health care renunciation for economic reasons in Switzerland. Swiss Med Wkly. 2011;141:w13165.

2 Coutaz M, Morisod J. Quelle perception les personnes âgées ont-elles des coûts médicaux qu'elles induisent? Rev Med Suisse. 2007;3:1912-5.

3 Newhouse JP, Manning WG, Morris CN, Orr LL, Duan N, Keeler EB, Leibowitz A, Marquis KH, Marquis MS, Phelps CE, Brook RH. Some interim results from a controlled trial of cost sharing in health insurance. N Engl J Med. 1981;305:1501-7.

4 Trivedi AN, Moloo H, Mor V. Increased ambulatory care copayments and hospitalizations among the elderly. N Engl J Med. 2010;362:320-8. 